\title{
Nonlinear eigenvalue problems for fourth order ordinary differential equations
}

\author{
by Jolanta PRzybycin (Kraków)
}

\begin{abstract}
This paper was inspired by the works of Chiappinelli ([3]) and Schmitt and Smith $([7])$. We study the problem $\mathcal{L} u=\lambda a u+f\left(\cdot, u, u^{\prime}, u^{\prime \prime}, u^{\prime \prime \prime}\right)$ with separated boundary conditions on $[0, \pi]$, where $\mathcal{L}$ is a composition of two operators of Sturm-Liouville type. We assume that the nonlinear perturbation $f$ satisfies the inequality $\left|f\left(x, u, u^{\prime}, u^{\prime \prime}, u^{\prime \prime \prime}\right)\right| \leq$ $M|u|$. Because of the presence of $f$ the considered equation does not in general have a linearization about 0. For this reason the global bifurcation theorem of Rabinowitz ([5], [6]) is not applicable here. We use the properties of Leray-Schauder degree to establish the existence of nontrivial solutions and describe their location. The results obtained are similar to those proved by Chiappinelli for Sturm-Liouville operators.
\end{abstract}

Let $\mathcal{L}$ be a differential operator of the form $\mathcal{L}=\mathcal{L}_{1} \circ \mathcal{L}_{0}$, where $\mathcal{L}_{i}$ denotes the Sturm-Liouville operator defined by $\mathcal{L}_{i} u=-\left(p_{i} u^{\prime}\right)^{\prime}+q_{i} u, i=0,1$. As usual we assume $p_{i} \in C^{3-2 i}[0, \pi], q_{i} \in C^{2-2 i}[0, \pi]$ and $p_{i}>0, q_{i} \geq 0$ on $[0, \pi]$. We denote by (B.C.) either the boundary conditions

$$
u(0)=u(\pi)=\mathcal{L}_{0} u(0)=\mathcal{L}_{0} u(\pi)=0
$$

or the boundary conditions

$$
u(0)=u(\pi)=u^{\prime}(0)=u^{\prime}(\pi)=0 .
$$

Let $a$ be a strictly positive continuous function on $[0, \pi]$. We assume that the operator $\mathcal{L}$ is symmetric and positive definite (which is satisfied in particular when $\left.\mathcal{L}_{0}=\mathcal{L}_{1}\right)$. Then the linear problem $\mathcal{L} v=$ aav in $(0, \pi)$ together with the boundary conditions (B.C.) has an increasing sequence of eigenvalues $0<\mu_{1}<\mu_{2}<\ldots$ with $\lim _{k \rightarrow \infty} \mu_{k}=\infty$. Each $\mu_{k}$ is simple (Bochenek [2]).

Now consider the equation

$$
\mathcal{L} u=\lambda a u+f\left(\cdot, u, u^{\prime}, u^{\prime \prime}, u^{\prime \prime \prime}\right) \quad \text { in }(0, \pi)
$$

1991 Mathematics Subject Classification: Primary 34B15.

Key words and phrases: bifurcation point, bifurcation interval, Leray-Schauder degree, characteristic value. 
together with the boundary conditions (B.C.). Assume that the nonlinear function $f$ is continuous on $[0, \pi] \times \mathbb{R}^{4}$ and satisfies

$$
\exists_{M>0} \forall(x, \xi, \eta, \gamma, \zeta) \in[0, \pi] \times \mathbb{R}^{4} \quad|f(x, \xi, \eta, \gamma, \zeta)| \leq M|\xi| .
$$

By a solution of (1) we understand a pair $(\lambda, u) \in \mathbb{R} \times\left(C^{4}[0, \pi] \cap\right.$ (B.C.)) satisfying (1).

Let $E=C^{3}[0, \pi] \cap$ (B.C.). It is a Banach space equipped with its usual norm, $\|u\|_{3}:=\|u\|_{0}+\left\|u^{\prime}\right\|_{0}+\left\|u^{\prime \prime}\right\|_{0}+\left\|u^{\prime \prime \prime}\right\|_{0}$, where $\|u\|_{0}=\sup _{x \in[0, \pi]}|u(x)|$.

Notice that it is sufficient to search for solutions of (1) in $\mathbb{R} \times E$.

Namely, by using the Green function $g$ of $\mathcal{L}$ together with the boundary conditions (B.C.), equation (1) can be converted into an equivalent integral equation in $\mathbb{R} \times E$ :

$$
u(\cdot)=\int_{0}^{\pi} g(\cdot, y)\left[\lambda a(y) u(y)+f_{u}(y)\right] d y=\lambda L u+F(u),
$$

where

$$
\begin{gathered}
L u=\int_{0}^{\pi} g(\cdot, y) a(y) u(y) d y, \quad F(u)=\int_{0}^{\pi} g(\cdot, y) f_{u}(y) d y, \\
f_{u}(y)=f\left(y, u(y), u^{\prime}(y), u^{\prime \prime}(y), u^{\prime \prime \prime}(y)\right) .
\end{gathered}
$$

Clearly $F: E \rightarrow E$ is continuous, and $L: E \rightarrow E$ is compact and linear.

Let $B=\left\{u \in E:\|u\|_{3} \leq \delta\right\}$. To verify that $F$ is compact we prove that $F(B)$ is relatively compact. For $u \in B$ we obtain the estimate

$$
\|F(u)\|_{3}=\sum_{i=0}^{3} \sup _{x \in[0, \pi]}\left|\int_{0}^{\pi} \frac{\partial^{i} g}{\partial x^{i}}(x, y) f_{u}(y) d y\right| \leq c \pi M\|u\|_{3} \leq c \pi M \delta,
$$

where $c$ depends on bounds for $g$ and $\partial^{i} g / \partial x^{i}$. Hence $F(B)$ is bounded in $E$. Moreover, $w=F(u)$ satisfies

$$
\mathcal{L} w=f\left(\cdot, u, u^{\prime}, u^{\prime \prime}, u^{\prime \prime \prime}\right) .
$$

Hence, solving the above equation for $w^{(4)}$ we obtain uniform bounds for the fourth derivatives of $F(u)$ in $B$. Applying the Arzelà-Ascoli theorem we deduce at once the compactness of $F$.

Notice that the eigenvalues $\mu_{k}$ of $\mathcal{L}$ are equal to the characteristic values of $L$ (i.e. there exist $v_{k} \in E, v_{k} \neq 0$, such that $v_{k}=\mu_{k} L v_{k}$ ). We denote by $r(L)$ the set of characteristic values of $L$.

Now we give a description of the spectrum for (1).

THEOREM 1. If $(\lambda, u)$ is a nontrivial solution of (1), then

$$
\lambda \in \bigcup_{k=1}^{\infty}\left[\mu_{k}-M / a_{0}, \mu_{k}+M / a_{0}\right]
$$

where $a_{0}=\min _{x \in[0, \pi]} a(x)$. 
Pr o of. The pair $(\lambda, u)$ satisfies (3). Multiplying both sides of (3) by $a^{1 / 2}$ we obtain

$$
\widehat{u}=\lambda H \widehat{u}+a^{1 / 2} F(u)
$$

where

$$
\widehat{u}=a^{1 / 2} u, \quad H \widehat{u}=\int_{0}^{\pi} a(\cdot)^{1 / 2} g(\cdot, y) a(y)^{1 / 2} \widehat{u}(y) d y .
$$

It is clear that $H$ is a selfadjoint operator on $L^{2}[0, \pi]$ and the set of characteristic values of $H, r(H)$, equals $r(L)$. For $\lambda \neq \mu_{k}, I-\lambda H$ is invertible, so that (4) is equivalent to

$$
\widehat{u}=(I-\lambda H)^{-1}\left(a^{1 / 2} F(u)\right) .
$$

We have

$$
a^{1 / 2} F(u)=\int_{0}^{\pi} a(\cdot)^{1 / 2} g(\cdot, y) a(y)^{1 / 2} \frac{f_{u}(y)}{a(y)^{1 / 2}} d y=H\left(a^{-1 / 2} f_{u}\right)
$$

and

$$
\left\|a^{-1 / 2} f_{u}\right\|_{L^{2}}^{2} \leq \int_{0}^{\pi} \frac{M^{2}}{a(y)} u(y)^{2} d y=\int_{0}^{\pi} \frac{M^{2}}{a(y)^{2}} \widehat{u}(y)^{2} d y \leq \frac{M^{2}}{a_{0}^{2}}\|\widehat{u}\|_{L^{2}}^{2} .
$$

Hence

$$
\|\widehat{u}\|_{L^{2}} \leq\left\|(I-\lambda H)^{-1} H\right\| \cdot\left\|a^{-1 / 2} f_{u}\right\|_{L^{2}} \leq\left\|(I-\lambda H)^{-1} H\right\| \frac{M}{a_{0}}\|\widehat{u}\|_{L^{2}} .
$$

Since $\left\|(I-\lambda H)^{-1} H\right\|^{-1}=\operatorname{dist}(\lambda, r(H))$ (Kato [4], p. 273) we conclude that $\operatorname{dist}(\lambda, r(L)) \leq M / a_{0}$ and the proof is complete.

From now on we assume additionally that

$$
\mu_{k}-\mu_{k-1} \rightarrow \infty \quad \text { as } k \rightarrow \infty
$$

This condition seems to be not particularly restrictive. Look at some examples.

EXAMPLE 1. If $\mathcal{L}_{0}, \mathcal{L}_{1}$ commute then the eigenvalues of the problem $\mathcal{L} u=\mu u$ subject to $u(0)=u(\pi)=\mathcal{L}_{0} u(0)=\mathcal{L}_{0} u(\pi)=0$ are of the form $\mu_{k}=\mu_{k}^{1} \mu_{k}^{0}$, where $\mu_{k}^{i}$ denotes the eigenvalue of $\mathcal{L}_{i}$. It is simple to show that (5) is satisfied since $\mu_{k}^{i}-\mu_{k-1}^{i} \rightarrow \infty$ as $k \rightarrow \infty, i=0,1$.

EXAmple 2. Consider the equation $u^{(4)}=\mu u$ with the boundary conditions $u(0)=u(\pi)=u^{\prime}(0)=u^{\prime}(\pi)=0$. It is easy to compute that the eigenvalues $\mu_{k}$ satisfy the condition $\sqrt[4]{\mu_{k}}-\sqrt[4]{\mu_{k-1}} \rightarrow 1$ as $k \rightarrow \infty$ and consequently we have (5).

The assumption (5) implies that, given any $c>0$, the intervals $\left[\mu_{k}-\right.$ $\left.c, \mu_{k}+c\right]$ are disjoint for $k$ large enough.

We can now formulate the main result. 
THEOREM 2. Let $k_{0}=\min \left\{\bar{k} \in \mathbb{N}: \mu_{k}-\mu_{k-1}>2 M / a_{0}\right.$ for $\left.k>\bar{k}\right\}$. Then for every $k>k_{0}$ and $\delta>0$ there exists a solution $(\lambda, u)$ of (1) with $\|u\|_{3}=\delta$ and $\lambda \in\left[\mu_{k}-M / a_{0}, \mu_{k}+M / a_{0}\right]$.

Proof. Let $B=\left\{u \in E:\|u\|_{3} \leq \delta\right\}$. Now fix $k>k_{0}$ and choose $\varepsilon>0$ such that

$$
\begin{aligned}
& \underline{\lambda}:=\mu_{k}-\left(M / a_{0}+\varepsilon\right)>\mu_{k-1}+\left(M / a_{0}+\varepsilon\right), \\
& \bar{\lambda}:=\mu_{k}+\left(M / a_{0}+\varepsilon\right)>\mu_{k+1}-\left(M / a_{0}+\varepsilon\right) .
\end{aligned}
$$

It is easy to see that

$$
\operatorname{dist}(\underline{\lambda}, r(L))=\operatorname{dist}(\bar{\lambda}, r(L))=M / a_{0}+\varepsilon .
$$

We argue by contradiction, so assume that $u \neq \lambda L u+F(u)$ for all $u \in \partial B$ and $\lambda \in[\underline{\lambda}, \bar{\lambda}]$. Since $\lambda L+F$ is compact on $B$, the Leray-Schauder degree of $\Phi(\lambda)=I-\lambda L-F$ with respect to $B$ and the point 0 is well defined for all $\lambda \in[\underline{\lambda}, \bar{\lambda}]$. By the homotopy invariance of the degree we get

$$
d(\Phi(\lambda), B, 0)=\text { const } \quad \text { for } \lambda \in[\underline{\lambda}, \bar{\lambda}] .
$$

In particular, we have

$$
d(\Phi(\underline{\lambda}), B, 0)=d(\Phi(\bar{\lambda}), B, 0) .
$$

Consider now the first term in (7). Notice that $u \neq \underline{\lambda} L u+t F(u)$ for $u \in \partial B$ and $t \in[0,1]$. If not, proceeding as in the proof of Theorem 1 , we obtain dist $(\underline{\lambda}, r(L)) \leq M / a_{0}$, which contradicts (6). So, using the homotopy invariance again we obtain

$$
d(\Phi(\underline{\lambda}), B, 0)=d(I-\underline{\lambda} L, B, 0)=i(\underline{\lambda})=(-1)^{\underline{\beta}},
$$

where $\beta$ is the sum of the multiplicities of the characteristic values of $\underline{\lambda} L$ in $(0,1)$. The same argument can be used for $\bar{\lambda}$, so that

$$
d(\Phi(\bar{\lambda}), B, 0)=i(\bar{\lambda})=(-1)^{\bar{\beta}} .
$$

The $\bar{\beta}$ sum differs from the $\beta$ sum by a term equal to the multiplicity of the characteristic value $\mu_{k} / \bar{\lambda}$ of $\bar{\lambda} L$. Since this is just the multiplicity of $\mu_{k}$ and $\mu_{k}$ is simple, $i(\underline{\lambda})=-i(\bar{\lambda}) \neq 0$ contrary to $(7)$. The theorem is proved.

Following Berestycki ([1]), by a bifurcation interval we understand an interval which contains at least one bifurcation point.

Let us mention an important consequence of Theorem 2.

Remark 3. For every $k>k_{0},\left[\mu_{k}-M / a_{0}, \mu_{k}+M / a_{0}\right] \times\{0\}$ is a bifurcation interval for (1).

EXAmple 3. Consider

$$
u^{(4)}=\lambda u+|u|
$$


in $(0, \pi)$ with the boundary conditions $u(0)=u(\pi)=u^{\prime \prime}(0)=u^{\prime \prime}(\pi)=0$. The equation $(*)$ has the family of solutions $\left(\lambda_{\gamma}, u_{\gamma}\right)=\left(k^{4}-\operatorname{sgn} \gamma, \gamma \sin k x\right) \in$ $\bigcup_{k=1}^{\infty}\left[k^{4}-1, k^{4}+1\right] \times E$. It is clear that all bifurcation points for $(*)$ are of the form $\left(k^{4}-1,0\right)$ or $\left(k^{4}+1,0\right)$.

ExAmple 4. Consider

$$
u^{(4)}=\lambda u+u \sin \left(u^{\prime \prime 2}+u^{\prime \prime \prime 2}\right)^{-1 / 2}
$$

in $(0, \pi)$ with $u(0)=u(\pi)=u^{\prime \prime}(0)=u^{\prime \prime}(\pi)=0$. Let $k=1$. We have the family of solutions $\left(\lambda_{\gamma}, u_{\gamma}\right)=(1-\sin (1 /|\gamma|), \gamma \sin x) \in[0,2] \times E$. All the points of the interval $[0,2] \times\{0\}$ are bifurcation points for $(* *)$.

\section{References}

[1] H. Berestycki, On some Sturm-Liouville problems, J. Differential Equations 26 (1977), 375-390.

[2] J. Bochenek, Nodes of eigenfunctions of certain class of ordinary differential equations of the fourth order, Ann. Polon. Math. 29 (1975), 349-356.

[3] R. Chiappinelli, On eigenvalues and bifurcation for nonlinear Sturm-Liouville operators, Boll. Un. Mat. Ital. (6) 4-A (1985), 77-83.

[4] T. Kato, Perturbation Theory for Linear Operators, Springer, Berlin, 1966.

[5] J. Przybycin, Some applications of bifurcation theory to ordinary differential equations of the fourth order, Ann. Polon. Math. 53 (1991), 153-160.

[6] P. H. Rabinowitz, Some aspects of nonlinear eigenvalue problems, Rocky Mountain J. Math. 3 (1973), 161-202.

[7] K. Schmitt and H. L. Smith, On eigenvalue problems for nondifferentiable mappings, J. Differential Equations 33 (1979), 294-319.

INSTITUTE OF MATHEMATICS

ACADEMY OF MINING AND METALLURGY

AL. MICKIEWICZA 30

30-059 KRAKÓW, POLAND 